\title{
Photoelectron Spin Polarization Approaching Unity: Photoionization of TI
}

\author{
N. Böwering, M. Salzmann, M. Müller, H.-W. Klausing and U. Heinzmann \\ Fakultät für Physik, Universität Bielefeld, D-4800 Bielefeld, F.R.G.; and Fritz-Haber-Institut der MPG, D-1000 Berlin 33, F.R.G.
}

Received July 16, 1989; accepted November 11, 1989

\begin{abstract}
It is shown that a complete photoelectron-spin polarization occurs independent of emission angle for two special sets of values of the dynamical photoionization parameters. In spin- and angle-resolved photoelectron spectroscopy of thallium the dynamical parameters were measured near the autoionization resonances at $149 \mathrm{~nm}$ and $82 \mathrm{~nm}$. A pronounced angular variation of the polarization component $A(\theta)$ was observed at $83 \mathrm{~nm}$. For both resonances the parameters were found to approach different limiting values such that the length of the spin-polarization vector assumes values close to unity at all emission angles. The implications of these results for the corresponding dipole transition matrix elements are discussed.
\end{abstract}

\section{Introduction}

Photoelectron-spin polarization of atoms can be studied using circularly polarized light and a Mott detector (for a review, see Ref. [1]). The experimental methods have advanced to include energy-, angle- and spin-resolved measurements of the photoelectrons emitted [2]. Complete polarization was considered by Brehm [3]. In a few cases, a complete polarization of the photoelectrons was observed or predicted at certain wavelengths: in the Fano effect of cesium [4] and for resonances in the photoionization of thallium $[5,6]$ and other atoms [7].

It is the purpose of this study to consider two cases of atomic photoionization where the limit of complete polarization of the photoelectrons at all emission angles is approached. In a first paragraph the two limiting cases are derived from the general expressions for the spin-polarization vector. Then, experimental data are presented for two autoionization resonances in the photoionization of thallium where such limiting cases are found to be approached. The consequences of these results on the corresponding dipole transition matrix elements are outlined in the discussion section.

\section{General relations}

For unpolarized atoms, the photoelectron emission process is characterized in general by five dynamical parameters: the partial cross section $\sigma$, the angular distribution parameter $\beta$ and the three spin-polarization parameters $A, \xi$ and $\alpha[1,7-9]$. The asymmetry parameters $\beta$ and $\alpha$ describe the dependence on emission angle $\theta$ for intensity and spin-polarization, respectively. $A$ is the angle-integrated value of the spinpolarization. The spin-polarization parameters characterize the three orthogonal components $A(\theta), P_{\perp}(\theta)$ and $P_{\mathrm{p}}(\theta)$ of the spin-polarization vector $\boldsymbol{P}(\theta)$. The reaction plane is given by the light propagation direction and the emission direction of the photoelectrons analysed. $P_{\perp}(\theta)$ is the component perpen- dicular to the reaction plane while $A(\theta)$ and $P_{\mathrm{p}}(\theta)$ are the components in the reaction plane parallel and perpendicular to the photon polarization, respectively.

For circularly polarized light these components are given by the following formulas [2, 7-9]:

$$
\begin{aligned}
A(\theta) & =\gamma \frac{\left[A-\alpha P_{2}(\cos \theta)\right]}{1-\beta / 2 P_{2}(\cos \theta)} \\
P_{\mathrm{p}}(\theta) & =\gamma \frac{-3 / 2 \alpha \sin \theta \cos \theta}{1-\beta / 2 P_{2}(\cos \theta)} \\
P_{\perp}(\theta) & =\frac{2 \xi \sin \theta \cos \theta}{1-\beta / 2 P_{2}(\cos \theta)}
\end{aligned}
$$

Here, $\gamma= \pm 1$ is the helicity of the light. With eqs. (1), (2) and (3), the length of the polarization vector can be obtained as a function of emission angle $\theta$ :

$$
\begin{aligned}
& |\boldsymbol{P}(\theta)| \\
& \quad=\left(\frac{\left[A-\alpha P_{2}(\cos \theta)\right]^{2}+\left(4 \xi^{2}+9 / 4 \alpha^{2}\right) \sin ^{2} \theta \cos ^{2} \theta}{\left[1-\beta / 2 P_{2}(\cos \theta)\right]^{2}}\right)^{1 / 2}
\end{aligned}
$$

where $P_{2}(\cos \theta)$ is the second Legendre polynomial. From this relationship we derive that the polarization vector can have a length of unity at all emission angles (i.e., $|\boldsymbol{P}|=1$ independent of $\theta$ ) for two special sets of values for the parameters:

case (a): $A=+1, \quad \alpha=\xi=\beta=0 ;$

case (b): $\quad A=-1 / 3, \quad \alpha=-4 / 3, \quad \xi=\beta=0$.

The solutions with reversed sign for case (a) and (b) might also occur in principle, but can be excluded if in addition it is required that

$1 \geqslant A \geqslant-1 / 2$ and $2 / 3 \geqslant \alpha \geqslant-4 / 3$.

This condition for $A$ can be derived from the relativistic formulas (5.64) and (5.65) of Huang [8] valid for the most common case of ionization of atoms with a filled electron shell in the initial atomic or final ionic state. The two cases (a) and (b) were also listed by Brehm [3] at a time when the existence of $\xi$ and thus $P_{\perp}(\theta)$ was not yet known.

Both in case (a) and (b) the polarization vector lies in the reaction plane, since $\xi$ and thus $P_{\perp}(\theta)$ are equal to zero. In addition, the angular intensity distribution is isotropic. In case (a) the polarization vector of unit length points in the light propagation direction. It is a trivial case, because $A$ can also be obtained in an angle-integrated experiment where all electrons are extracted regardless of their emission direction [4]. Here, independent of $\theta$, the component $A(\theta)$ is constant 

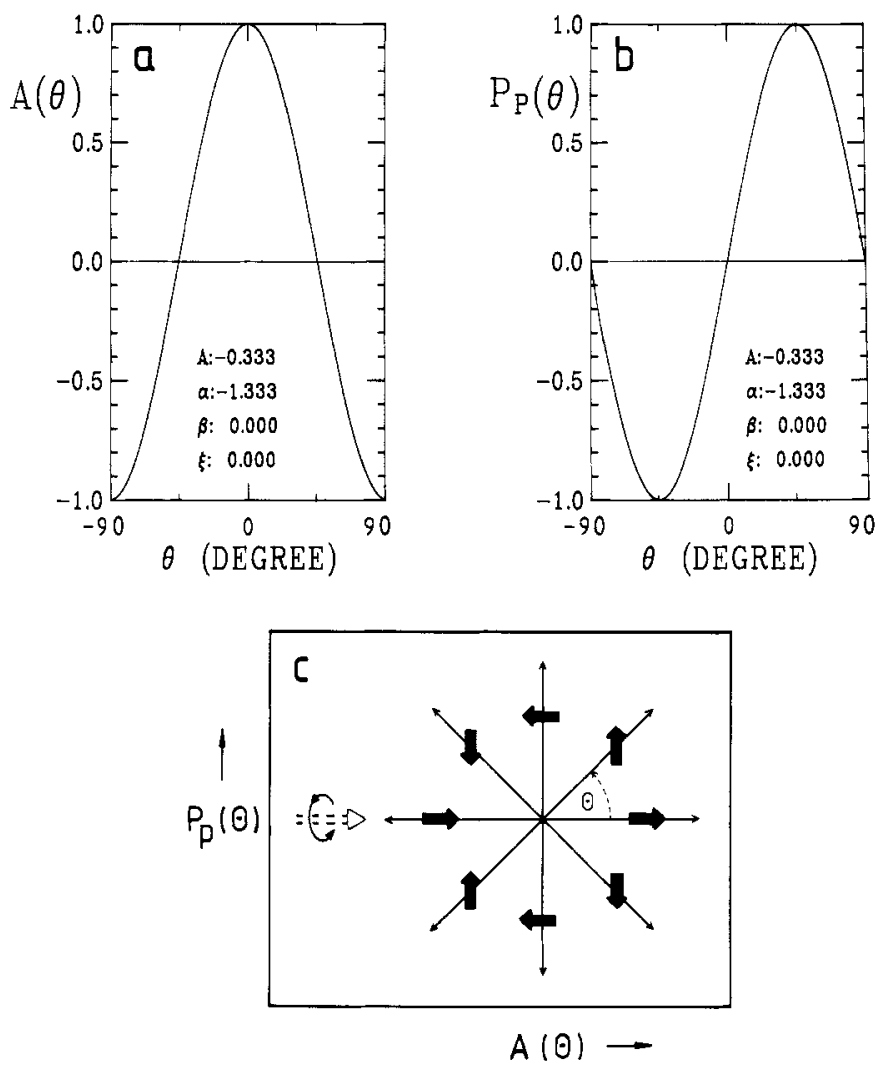

Fig. 1. Spin-polarization vector for complete polarization at all emission angles, case (b). (a) Angular dependence for the component $A(\theta)$. (b) Angular dependence for the component $P_{\mathrm{p}}(\theta)$. (c) Spin-polarization vector (solid arrows) in the reaction plane [spanned by $A(\theta)$ and $P_{\mathrm{p}}(\theta)$ ] for different emission angles $\theta$. Right-handed circularly polarized light is incident from the left.

and unity while the other two components $P_{\mathrm{p}}(\theta)$ and $P_{\perp}(\theta)$ vanish. Case (b), where the angular dependence of the polarization vector is more complicated, is illustrated in Fig. 1. Here both components $A(\theta)$ and $P_{\mathrm{p}}(\theta)$ vary from +1 to -1 , but shifted in phase by 45 degrees whereas $P_{\perp}(\theta)$ is zero. Nevertheless, the total polarization is complete although its angle-integrated value $A$ is not equal to unity. With increasing photoelectron emission angle $\theta$ the polarization vector revolves twice as much and points in the direction given by $2 \theta$. This is illustrated in Fig. 1(c).

\section{Experimental}

The photoemission measurements were performed energy-, angle- and spin-resolved with out apparatus at the storage ring BESSY using circularly polarized synchrotron radiation (for a description of the apparatus, see Ref. [2]). Briefly, the VUV radiation emitted out of plane is dispersed by a $6.5 \mathrm{~m}$ normal incidence monochromator $(\Delta \lambda=0.5 \mathrm{~nm})[10]$ and crosses an atomic beam of thallium originating from a resistively heated oven. The photoelectrons emitted at angle $\theta$ are energy-analyzed by a rotatable electron spectrometer ( $\Delta \theta= \pm 5$ degrees acceptance angle). Subsequently, the electrons are accelerated to $100 \mathrm{keV}$ and scattered off a gold foil in a Mott detector (Sherman function: -0.21 ) for spinpolarization analysis. The two transverse polarization components $A(\theta)$ and $P_{\perp}(\theta)$ can be determined simultaneously. From a measurement of the angular distribution of these two components all dynamical parameters (except for the absolute value of the cross-section $\sigma$ ) can be determined. The par- ameters $A$ and $\xi$ can also be obtained from a measurement of $A(\theta)$ at the magic angle $\theta_{\mathrm{m}}=54.7^{\circ}$. The component $P_{\mathrm{p}}(\theta)$ can not be detected directly in our geometry; however, it contains only redundant information, since $\alpha$ and $\beta$ are also contained in $A(\theta)$ [see eq. (1)].

\section{Results}

At the evaporation temperatures used $(1000 \mathrm{~K})$ almost all atoms are in the $\mathrm{Tl}\left(5 d^{10} 6 s^{2} 6 p\right)^{2} P_{1 / 2}$ ground state. In this study we report on measurements of the dynamical photoionization parameters near the $\mathrm{Tl}\left(5 d^{10} 6 s 6 p^{2}\right)(J=1 / 2)$ and $\operatorname{Tl}\left(5 d^{9} 6 s^{2} 6 p^{2}\right)(J=3 / 2)$ resonances at around $\lambda=149 \mathrm{~nm}$ and $\lambda=82 \mathrm{~nm}$, respectively, for the $\mathrm{Tl}^{+}\left(5 d^{10} 6 s^{2}\right){ }^{1} S_{0}$ final ionic state ( $\varepsilon s_{1 / 2}$ and $\varepsilon d_{3 / 2}$ outgoing partial waves).

At $\lambda=149 \mathrm{~nm}$ the cross section is very large and the autoionization resonance dominates the spectrum entirely. Previously, in an angle-integrated measurement of the parameter $A$ at $149 \mathrm{~nm}$ a value of 0.5 was obtained, limited by the radiation bandwidth used [11]. Later, the polarization parameter $A$ was predicted to be nearly unity at the peak of the resonance in an RPAE calculation by Cherepkov $[6,12]$. This corresponds to case (a). Recently, we have measured a value of $A=A\left(\theta_{\mathrm{m}}\right)=(0.92 \pm 0.05)$ and found that $\alpha, \zeta$ and $\beta$ are zero at $148.9 \mathrm{~nm}$ within the experimental error limits. This is in excellent agreement with the theoretical prediction $[6,12]$ if the experimental resolution of $\Delta \lambda=0.5 \mathrm{~nm}$ is taken into account [5]. In addition, for photoelectrons emitted in the forward direction a value of $A(\theta=0)=(1.00 \pm 0.05)$ was measured directly. This is also required from general theoretical considerations for this final ionic state $[1,3,13]$. The wavelength dependence of the parameters in this resonance and the comparison with the theoretical curve will be published elsewhere [5].

For the resonance near $82 \mathrm{~nm}$ no prediction exists. At this wavelength the final ionic states $\mathrm{Tl}^{+}\left(5 d^{10} 6 s 6 p\right){ }^{3} P_{0},{ }^{3} P_{1}$, and ${ }^{3} P_{2}$ are also accessible in addition to the $\mathrm{Tl}^{+}\left(5 d^{10} 6 s^{2}\right){ }^{1} S_{0}$ state. They can be studied separately by means of the electron spectrometer. For the $\mathrm{Tl}^{+}{ }^{1} S_{0}$ channel the relative cross

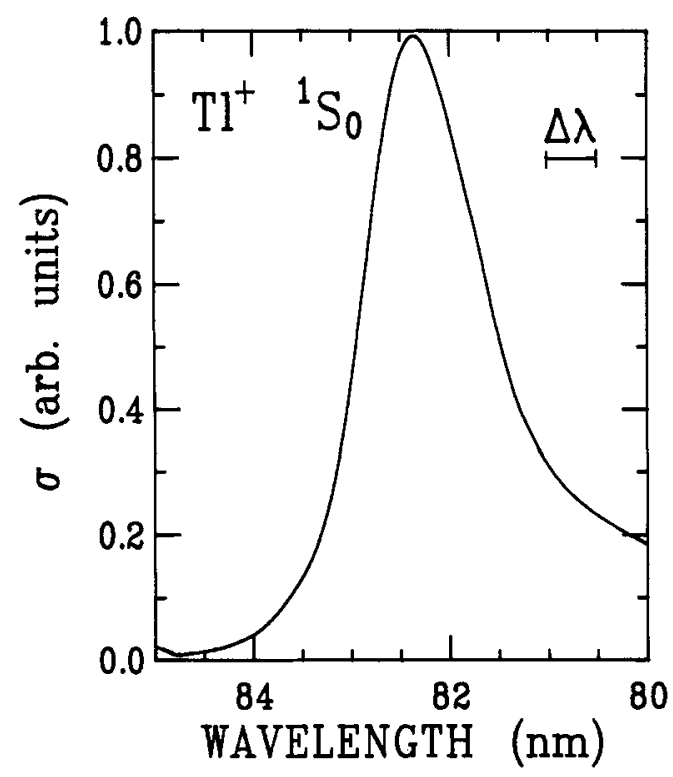

Fig. 2. Relative partial cross section for the $\mathrm{Tl}\left(5 d^{9} 6 s^{2} 6 p^{2}\right)(5 / 2,2)_{3 / 2}$ autoionization resonance, $\mathrm{Tl}^{+}\left(5 d^{10} 6 s^{2}\right){ }^{1} S_{0}$ final ionic state. The assignment is according to Ref. [15]. 


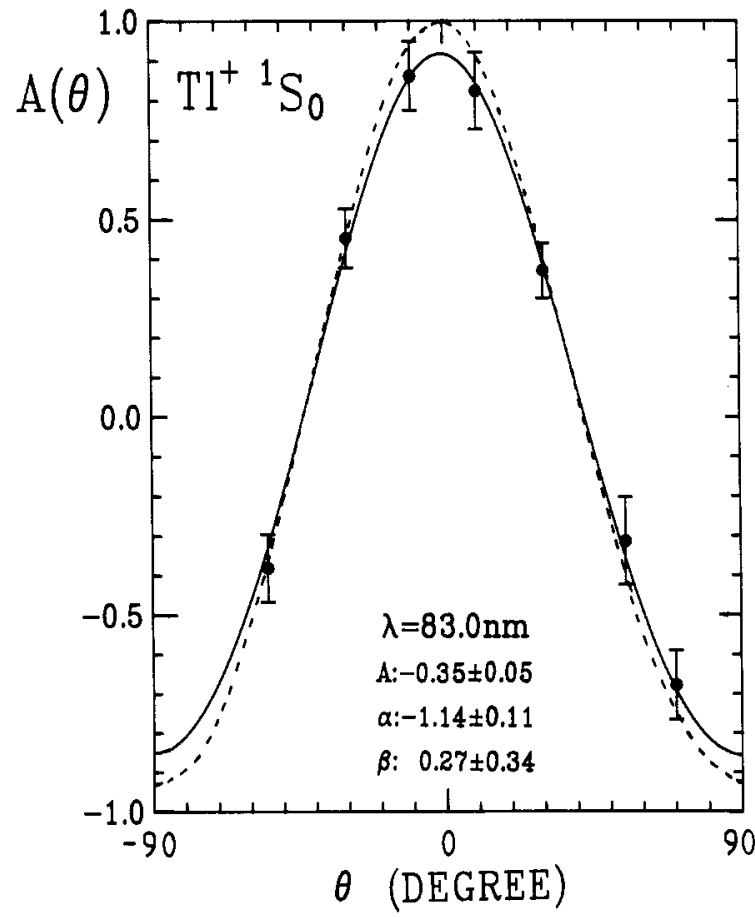

Fig. 3. Angular dependence for the spin-polarization component $A(\theta)$ at $\lambda=83 \mathrm{~nm}, \sigma^{+}$-light. The solid line drawn through the data points represents a least-squares fit yielding the parameters given in the figure. The dashed curve is explained in the text.

section measured at the magic angle is shown in Fig. 2. An asymmetric profile with a deep minimum at $84.5 \mathrm{~nm}$ is observed. This resonance was also investigated by Connerade and coworkers [14-16] with high resolution in absorption (sum of all four channels) and assigned to an overlap of $\mathrm{Tl}$ $\left(5 d^{9} 6 s^{2} 6 p^{2}\right)(5 / 2,2) J=3 / 2$ and $J=1 / 2$, with the $J=3 / 2$ resonance dominating at the long wavelength side. In addition, these studies revealed that weak interloping Rydberg series converging to the $\mathrm{Tl}^{+1} P_{1}$ state exist. We have also measured the spin-polarization parameters and the $\beta$ parameter for this resonance.

The angular dependence for the component $A(\theta)$ at $\lambda=83 \mathrm{~nm}$ is shown in Fig. 3. At this wavelength the most pronounced distribution was observed with large negative values for emission perpendicular to the photon direction. The solid line represents the result of a least-squares fit to the data. The parameters resulting from the fit are given in the figure. This fit curve does not go through unity at zero degrees as required [3]. This can be due to the finite angular acceptance of the spectrometer which causes the data points at 10 degrees to be lowered. In order to eliminate resolution effects in an approximate way, we have also plotted a dashed curve which fulfills the requirement mentioned above and is obtained by dividing the data of the full curve by 0.92 . At any rate, the data obtained are compatible with a large variation of $A(\theta)$ at this particular wavelength from values of almost 1.0 at 0 degrees to -0.9 at 90 degrees.

At other wavelength positions within the resonance, we have also obtained angular distributions for $A(\theta)$ (which are less pronounced) and the parameter $\xi$. The results for the dependence on photon wavelength of the spin-polarization parameters $A$ and $\xi$, and the asymmetry parameters $\alpha$ (for the spin polarization) and $\beta$ (for the intensity distribution) are shown in Fig. 4. With increasing wavelength the parameters approach the limiting values of case (b) outlined above as can
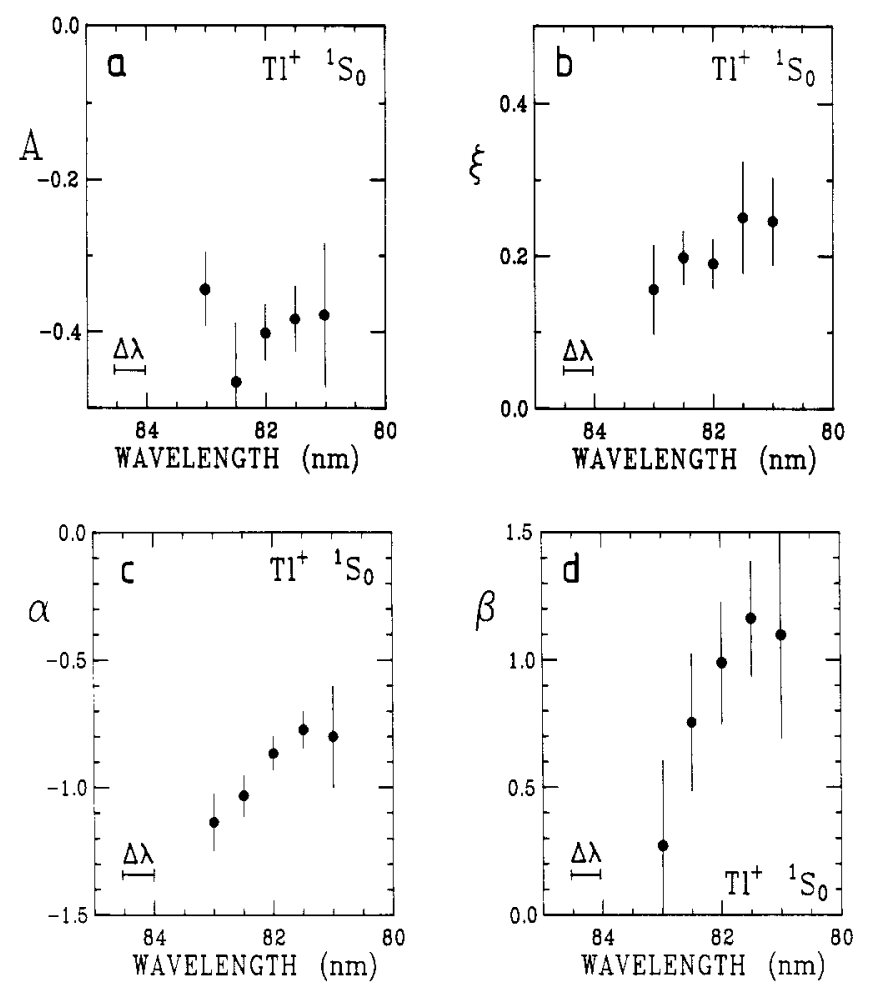

Fig. 4. (a-d) Wavelength dependence of the spin-polarization parameters $A$, $\zeta$ and $\alpha$ and of the angular asymmetry parameter $\beta, \mathrm{Tl}^{+}\left(5 d^{10} 6 s^{2}\right){ }^{1} S_{0}$ final ionic state.

be seen most clearly from the variation of the asymmetry parameters obtained. However, the count rates were too low at above $\lambda=83 \mathrm{~nm}$ (see Fig. 2) to extend the measurements further. (At $83 \mathrm{~nm}$ the signal intensity was more than two orders of magnitude smaller than at $149 \mathrm{~nm}$ ).

In Fig. 5 the length of the spin-polarization vector according to eq. (4) is plotted for the parameters determined at $\lambda=83 \mathrm{~nm}$ as a function of $\theta$. The length of the polarization vector is near 0.9 at all emission angles. The deviation from unity is mainly caused by the fact that the measured parameter $\alpha$ differs considerably from its limiting value of $-4 / 3$. The experimental error limits are largest at 0 degrees and smallest at the magic angle where the error from the parameter $\beta$ does not contribute. The values for the dashed curve are for the corresponding values of Fig. 3 to indicate the

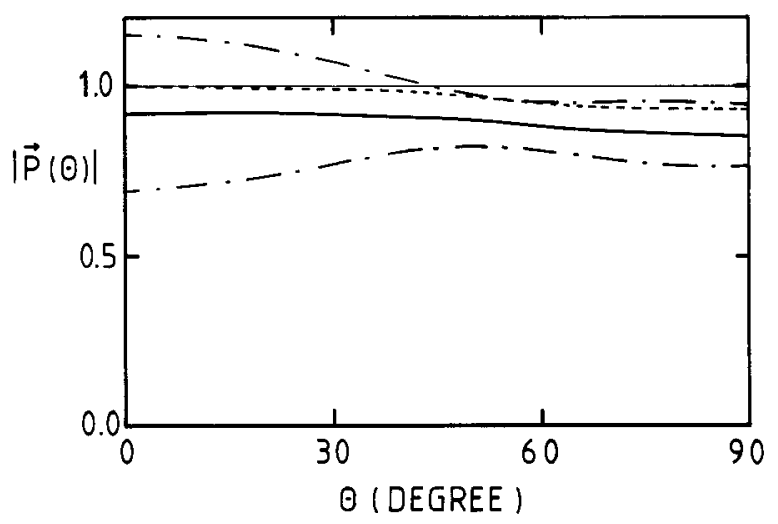

Fig. 5. Length of the spin-polarization vector as determined from the dynamical parameters measured at $\lambda=83 \mathrm{~nm}$. The dot-dashed lines indicate the error limits obtained by error propagation from the uncertainties of the various parameters. Only the first quadrant is shown; the values at other angles are determined by symmetry. The dashed curve corresponds to the dashed curve in Fig. 3. 
length of the polarization vector if the angular resolution would be perfect.

\section{Discussion}

The results described above show two different experimentally observed cases where the length of the polarization vector is near 0.9 or larger at all emission angles. The limiting values for the dynamical parameters which are approached experimentally are given by cases (a) and (b) [see eqs. (5) and (6)].

The results can be discussed in this limit with the help of the reduced dipole transition matrix elements $\left(D_{1 / 2}, D_{3 / 2}\right.$ and phase-shift difference $\delta_{1 / 2}-\delta_{3 / 2}$ ) relevant for this ionization channel. Table 1 of Ref. [17] gives the analytical dependence of the parameters on the matrix elements. Since the $\mathrm{Tl}^{+}{ }^{1} S_{0}$ final ionic state has filled electron shells, autoionization resonances with $J=3 / 2$ decay only with $\varepsilon d_{3 / 2}$ outgoing partial waves while resonances with $J=1 / 2$ are only associated with $\varepsilon s_{1 / 2}$ waves.

For pure case (a) with $A=1$, the $D_{3 / 2}$ transition amplitude vanishes (i.e., no outgoing $\varepsilon d_{3 / 2}$ partial wave) and the ionization process feeds the $\varepsilon s_{1 / 2}$ channel only. The complete spin polarization is due to the dominance of the $s_{1 / 2}$ partial wave. Our experimentally observed value for $A$ at $148.9 \mathrm{~nm}$ is still limited by the instrumental resolution. Due to the small but non-vanishing $\varepsilon d_{3 / 2}$ contribution at this photon energy the RPAE-calculation predicts $A$ to be smaller than unity, namely $A=0.998$ [12]. Likewise, $\alpha, \xi$ and $\beta$ are calculated to go through zero at slightly different photon energies [12].

In order to obtain pure case (b) the following special relationships are required to be fulfilled (see Table 1, Ref. [17]:

$D_{3 / 2}=-2 \sqrt{2} D_{1 / 2}$

and

$\delta_{1 / 2}-\delta_{3 / 2}=0$.

Both conditions have to be satisfied simultaneously at the same photon energy. In this limiting case, the two matrix elements are real and the intensity for the $\varepsilon d_{3 / 2}$ wave is 8 times larger than for $\varepsilon s_{1 / 2}$. Here the complete polarization occurs due to interference of the amplitudes of the outgoing partial waves leading to a suppression of a particular spin orientation in a similar fashion as in the Fano effect near a Cooper minimum of alkali atoms [18]. However, the latter effect corresponds to case (a) while here case (b) of complete polarization accompanied by a pronounced angular distribution applies.

For the resonance with peak at around $82 \mathrm{~nm}$ the $\varepsilon d_{3 / 2}{ }^{-}$ channel dominates, as can be seen from the large negative values of $A$ [Fig. 4(a)] in agreement with the assignment [15]. However, there are also weaker $(J=1 / 2)$-contributions in this wavelength region [14] and the resonance has a deep minimum at the long wavelength side (see Fig. 2) [19]. Apparently, this causes the $\varepsilon s_{1 / 2}$ channel to increase in strength relative to the $\varepsilon d_{3 / 2}$ channel in the wing of the resonance [reflected by the larger value of $A$ at $83 \mathrm{~nm}$ in Fig. 4(a)]. In addition, the parameter $\xi$ tends to small values (i.e., the phase-shift difference tends to zero) [see Fig. 4(b)] and case (b) is approached. However, since case (b) is not reached exactly, eqs. (8) and (9) are only fulfilled approximately. From the data of Fig. 4 one might extrapolate that the limiting case is even more closely approximated at around 83.5 to $84 \mathrm{~nm}$.

\section{Conclusion}

Two different particular cases were observed in the photoionization of thallim where a very high polarization of the photoelectrons occurs at all emission angles. The second case can only be observed in an angle-resolved experiment and is accompanied by a pronounced angular distribution for the spin-polarization component $A(\theta)$.

\section{Acknowledgement}

We express our thanks to the BESSY staff, in particular to F. Schäfers, for their help and acknowledge support by the BMFT under contract 05331 and 431 AX. We also thank N. Müller for illuminating discussions.

\section{References}

1. Heinzmann, U., Fundamental Processes in Atomic Collision Physics (Edited by H. Kleinpoppen, J. S. Briggs and H. O. Lutz), p. 269, Plenum (1985).

2. Heckenkamp, Ch., Schäfers, F., Schönhense, G. and Heinzmann, U., Z. Phys. D2, 257 (1986).

3. Brehm, B., Z. Phys. 242, 195 (1971).

4. Heinzmann, U., Kessler, J. and Lorenz, J., Phys. Rev. Lett. 25, 1325 (1970).

5. Müller, M., Böwering, N., Svensson, A. and Heinzmann, U., To be published in J. Phys. B.

6. Cherepkov, N. A., J. Phys. B10, L653 (1977).

7. Cherepkov, N. A., Adv. At. Mol. Phys. 19, 395 (1983).

8. Huang, K. N., Phys. Rev. A22, 223 (1980).

9. Several different notations are in use. A comparison of the polarization parameters introduced by several authors can be found in Table 1 of Raseev, G., Keller, F. and Lefebvre-Brion, H., Phys. Rev. A36, 4759 (1987).

10. Schäfers, F., Peatman, W., Eyers, A., Heckenkamp, Ch., Schönhense, G. and Heinzmann, U., Rev. Sci. Instrum. 57, 1032 (1986).

11. Heinzmann, U., Heuer, H. and Kessler, J., Phys. Rev. Lett. 34, 441 (1975).

12. Cherepkov, N. A., Opt. Spectrosc. 49, 582 (1980).

13. Cherepkov, N. A., Phys. Lett. 40A, 119 (1972).

14. Connerade, J. P., Proc. R. Soc. Lond. A362, 361 (1978).

15. Baig, M. A. and Connerade, J. P., J. Phys. B18, 3487 (1985).

16. Connerade, J. P., Lane, A. M. and Baig, M. A., J. Phys. B18, 3507 (1985).

17. Müller, M., Böwering, N., Schäfers, F. and Heinzmann, U., Physica Scripta 41, 42 (1990).

18. Fano, U., Phys. Rev. 178, 131 (1969).

19. The data might also be influenced by the weak $\mathrm{Tl}\left(5 d^{10} 6 s 6 p\right){ }^{\prime} P_{1} 9 p$ resonance at $83.4 \mathrm{~nm}$ observed only in the high resolution absorption spectra [14] if it has a contribution to the $\mathrm{Tl}^{+}{ }^{1} S_{0}$ channel. 